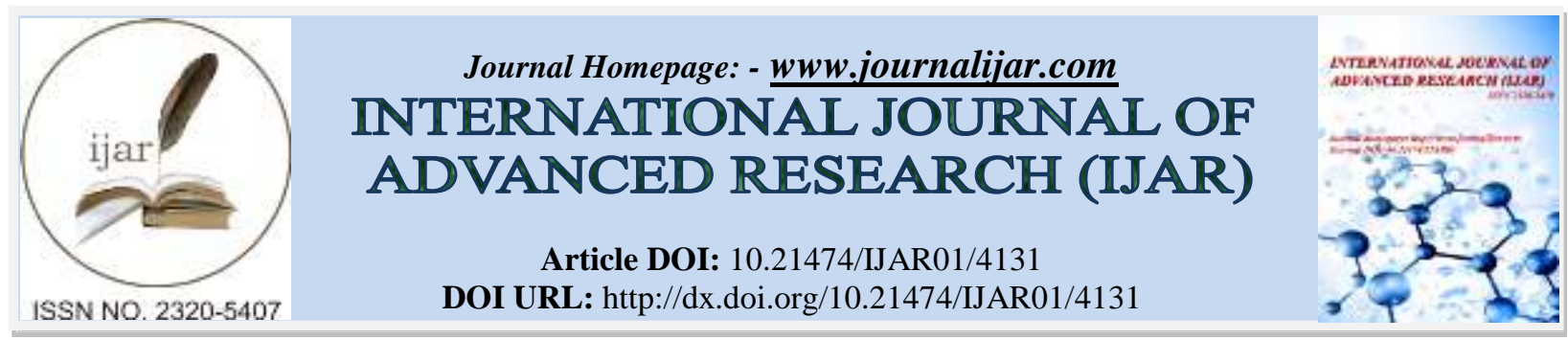

RESEARCH ARTICLE

\title{
POLYMORPHISMS OF ESTROGEN BIOSYNTHESIS AND METABOLIZING GENES IN EGYPTIAN WOMEN WITH BREAST CANCER.
}

\author{
"Wafaa Tolba1, Rizk El Baz², Azza I Othman ${ }^{3}$, Sameh Roshdy ${ }^{4}$ and Ahmad Settin'. \\ 1. PH D candidate, Physiology, Zoology Department, Faculty of science, Mansoura University; Egypt. \\ 2. Asst Professor, Molecular Biology, Genetics Unit, Mansoura University; Egypt. \\ 3. Professor, Physiology, Zoology Department, Faculty of science, Mansoura University; Egypt. \\ 4. Asst Professor, Department of General Surgery \& Oncology, Faculty of Medicine, Mansoura University; \\ Egypt. \\ 5. Professor, Molecular Biology, Genetics Unit, Mansoura University; Egypt.
}

\section{Manuscript Info}

[.........................

Manuscript History

Received: 04 March 2017

Final Accepted: 02 April 2017

Published: May 2017

Key words:-

CYP17; CYP1A1; CYP1B1; COMT;

Cancer breast; Egyptian women.

\section{Abstract}

Polymorphisms of genes responsible for biosynthesis and metabolism of estrogen including CYP and COMT groups might play a role in breast cancer. This study aims to investigate the association of CYP17 MspA1I, CYP1A1 MspI, CYP1B1 G>C, and COMT G>A polymorphisms with breast cancer in Egyptian women. Participants were in the form of 152 Egyptian women with cancer breast in addition to 100 healthy controls. They were subjected to DNA analysis using PCR-RFLP technique to characterize genetic polymorphisms of CYP17 MspA1I, CYP1A1 MspI, CYP1B1 G>C, and COMT G>A. Interestingly all these polymorphisms showed a positive association with cancer breast but in a variable degrees. Highest association was found with CYP1B1 C allele $(p=0.000, \mathrm{OR}=10.26,95 \% \mathrm{CI}=5.98-$ 17.8) followed by COMT A allele $(p=0.000, \mathrm{OR}=6.66,95 \% \mathrm{CI}=4.09$ - 10.9) then Cyp1A1 MspI C allele ( $p=0.000, \mathrm{OR}=4.46,95 \% \mathrm{CI}=2.68$ -7.47) and lastly the CYP17 MspA1 C allele ( $p=0.058, \mathrm{OR}=1.46,95 \%$ $\mathrm{CI}=1.0-2.1)$. Regarding clinical presentation, COMT A allele carriage was significantly higher among cases with positive lymph nodes $(\mathrm{p}=0.02)$ and in pre-menopausal cases $(p=0.020)$ while CYP 17 MspA1I C allele carriage was significantly higher among cases with negative breast feeding $(\mathrm{P}=0.043)$. We can come to a conclusion that rare alleles of estrogen biosynthesis and metabolizing genes particularly CYP1B1 G>C, and COMT G>A followed by CYP1A1 MspI, and CYP17 MspA1I are associated with breast cancer among Egyptian women.

Copy Right, IJAR, 2017,. All rights reserved.

\section{Introduction:-}

Breast tumors were described by the Egyptians then subsequently by Greeks and Romans since ancient times (Hosny, Elkaffas , 2002). Moreover, the most known cancer among women was breast cancer (BC), as per the Egyptian National Cancer Institute (NCI) series (Elatar, 2002). Known established BC risk factors include inherited mutations such as the genes of BRCA1 and BRCA2, estrogens lifelong exposure (late menopause and early 
menarche), alcohol, smoking, postmenopausal obesity in addition fatty diet (Fredslund et al.; 2012). Estrogen metabolism is affected by four possible pathways that might contribute to cancer evolution. These include the 16 alpha hydroxylation pathway, the pathway of 2-hydroxylation, the pathway of 4-hydroxylation through redox cycling, besides 4-hydroxyestradiolquinone- adenine/guanine adduct depurination pathway (yue, et al; 2013). Single nucleotide polymorphisms (SNPs) in genes of estrogen biosynthesis and metabolism might have an effect on levels of circulating estrogen and affect the susceptibility of breast cancer (Bozina, et al; 2009). The human CYP17 gene found on 10q24.3 chromosome (Fan, et al; 1992), encodes for cytochrome p450c17A that mediates activities vital for the endogenous steroid hormones production, such as estrogen and androgen (Miller, 1998). The most studied single nucleotide polymorphism (SNP) in CYP17 gene was a T to C (A1 to A2) substitution at 34 bp (base pair) upstream of the translation initiation site of CYP17 (rs 743572) (Cary et al; 1994). This substitution might create an additional SP1-type (CCACC box) binding site in promoter region, enhancing transcription, and expression of CYP17, hence an increased estrogen level (Carey, et al; 1994, Feigelson et al; 1998).

The Cytochrome P450 1A1 (CYP1A1) gene, located at 15q22-q24, (Kawajiri et al; 1986). It is used in estrone and estradiol conversion to 2-hydroxyestradiol (Cavalieri, et al; 2001). It also activates metabolism of polycyclic aromatic hydrocarbons to aryl epoxides by its aryl hydrocarbon hydroxylase activity (Law, 1990). A well-known single nucleotide polymorphism affecting this gene is the $6235 \mathrm{~T}>\mathrm{C}$ also known as CYP1A1*2A polymorphism in the 3'untranslated region, could be identified by Msp I restriction enzyme (Reding, et al; 2012). This polymorphism was more studied in association with risk of breast cancer (dos Santos, et al; 2011, da Fonte de Amorim, et al; 2002). The CYP1B1 gene is located at 2p21-p22 chromosome. It encodes for the main cytochrome p450 enzyme which catalyzing the formation of estrogen metabolite called 4-hydroxy estrogen, that has carcinogenic effect in animal models due to its high hormonal activity (Kristensen, et al; 2000). Moreover, 4-hydroxyestradiol can undergo redox cycling (Liehr, et al; 1986) that causes the formation of free radicals as well as reactive semiquinone/ quinone intermediates which are potentially noxious to the DNA (Cavalieri, et al; 1997). Val 432 Leu (G > C) polymorphism of the cytochrome p450 1B1 (CYP1B1) gene was 2.4- to 3.4-fold higher catalytically active than the wild type one (Mitrunen, Hirvonen, 2003).

Catechol-O-Methyltransferase enzyme (COMT) activates the O-methylation of 2- and 4-OHE2 (Yager, Liehr, 1996). COMT activity is found highly in kidney and liver, and it is also present at high levels in RBCs, brain, the mammary gland and uterine endometrium. The COMT gene, found on 22q11.1-q11.2 chromosome, has a G>A single-nucleotide polymorphism in codon 158/108 of the membrane bound/cytosolic form (Butterworth, Dragunow, 1996). It causes an amino acid change, Val>Met, which determining high- and low-activity alleles of the enzyme (Lachman, et al; 1996). It was proved that the COMT Met allele, the low-activity and heat-labile enzyme, was four to five fold less effective in catechol substrates methylation in vitro (Scanlon, et al;1979). The decreased COMT activity might cause accumulation of 4-OHE2 which may confer high breast cancer risk, however, the results remained controversial (He, et al; 2012).

This study is aiming to test for the association between polymorphisms of CYP17 MspA1I, CYP1A1 MspI, CYP1B1 G>C, and COMT G>A genes and breast cancer in Egyptian women.

\section{Materials and Methods:-}

This work is a case controlled study involving 152 Egyptian women with breast cancer admitted in the Oncology Center, College of Medicine, Mansoura University, Egypt. Breast cancer cases were compared to 100 unrelated healthy women which are age and locality matched. All these participants were exposed to thorough history analysis of items like age, socioeconomic level, education, work, nutrition, smoking habits, consanguinity, family history, parity, abortion, age at menarche, menopause, breast feeding, use of oral contraceptives, stage and grade of cancer, number of lymph nodes, estrogen receptors, progesterone receptors and site of metastases.

For all participants, DNA extraction was done from their peripheral bloods samples. Characterization of CYP17 MspA1I, CYP1A1 MspI, CYP1B1 G>C \& COMT G>A gene polymorphisms was done by suing PCR_RFLP (polymerase chain reaction, restriction fragment length polymorphism) technique as being described in table 1, fig $1-4$.

\section{Statistical Analysis:-}

SPSS program version 17 was used for analyzing the data statistically. Comparing genotype and allele frequencies among cases and controls was done using the Fisher's Exact and Chi square tests with the odds ratio (OR) and 95\% 
confidence intervals (95\% CI). Testing for genetic equilibrium was done using Hardy-Weinberg Equilibrium (HWE). p value $<0.05$ was considered significant.

\section{Results:-}

Interestingly all studied polymorphisms showed a positive association with cancer breast in Egyptian women but in variable degrees. Highest association was found with CYP1B1 C allele $(53.3 \%$ vs. $10.0 \%, p=0.000$, OR=10.26, $95 \% \mathrm{CI}=5.98-17.8)$ followed by COMT A allele $(51.0 \%$ vs. $13.0 \%, p=0.000$, OR=6.66, 95\% CI= $4.09-10.9)$ followed by Cyp1A1 MspI C allele (37.8 \% vs. $22.0 \%, p=0.000$, OR=4.46, 95\% CI=2.68 -7.47) and lastly the CYP17 MspA1I C allele (41.8\% vs. $33.0 \%, p=0.058, \mathrm{OR}=1.46,95 \% \mathrm{CI}=1.0-2.1)$ (tables 2-4). All these genes were positively associated with breast cancer in the dominant model, i.e. significant higher frequency of COMT $\mathrm{GA}+\mathrm{AA}(91.4 \%$ vs. $26.0 \%$; $=0.000, \mathrm{OR}=30.43,95 \% \mathrm{CI}=14.0-76.4) ; \mathrm{CYP} 1 \mathrm{~B} 1 \mathrm{GC}+\mathrm{CC}(73.1 \%$ vs. $20.0 \%$; $\mathrm{p}=0.000, \mathrm{OR}=10.8,95 \% \mathrm{CI}=5.66-20.87)$; higher frequency of CYP1A1 MspI TC+CC (56.6\% vs. $24.0 \% ; p=0.000$, $\mathrm{OR}=4.13,95 \% \mathrm{CI}=2.3-7.5)$ and $\mathrm{CYP} 17 \mathrm{MspA} 1 \mathrm{I} \mathrm{TC}+\mathrm{CC}(72.4 \%$ vs. $58.0 \%$; $\mathrm{P}=0.026, \mathrm{OR}=1.9,95 \% \mathrm{CI}=1.07-$ 3.34).

Comparing cases regarding the frequency of genotypes of CYP17 MspA1I, CYP1A1 MspI, Cyp1B1 G>C, and COMT G>A gene polymorphisms in relation to all studied demographic, clinical parameters and laboratory parameters (table 6), no significant association was found except for that of CYP 17 MspA1I C allele carriage $(\mathrm{CT}+\mathrm{CC})$ that was significantly higher among cases with negative breast feeding $(\mathrm{P}=0.043, \mathrm{OR}=6.474,95 \%$ $\mathrm{CI}=0.85-135.69)$ and COMT A allele carriage (GA+AA) that was significantly higher among cases with positive lymphadenopathy $\mathrm{p}=0.02, \mathrm{OR}=0.12,95 \% \mathrm{CI}=0.01-0.930$ and also in premenopausal cases compared to postmenopausal cases $(\mathrm{P}=0.020, \mathrm{OR}=0.190,95 \% \mathrm{CI}=0.028-0.959)$.

\section{Discussion:-}

This study, to our knowledge is the first report of testing the association of polymorphic genetic variants involved in biosynthesis and metabolism of estrogen with breast cancer among Egyptian women. The results showed a significant increase of the frequency of certain genotypes among patients compared to controls.

The highest degree of association was found with C allele carriage of CYP1B1 polymorphism. The same was reported among American CC homozygous women (Sigurdson, et al; 2009); Nigerian premenopausal women (Okobia, et al; 2009); Han Chinese women (Jiao, et al; 2010); Greenlandic Inut premenopausal women (Ghisari, et al; 2014). On the other hand, negative association was observed in other studies in American women (Reding, et al; 2009); German women (MARIE-GENICA, 2009); Polish women (Ociepa-Zawal, et al; 2009); Brazilian pre or postmenopausal women (dos Santos, et al; 2011); Caucasian Slovenian postmenopausal women (Cerne, et al; 2011); Nigerian postmenopausal women (Okobia, et al; 2009); Thai women (Sangrajrang, et al; 2009); Han Chinese women (Sun, et al; 2015). On the contrary, a reverse association with the $\mathrm{G}$; and not the $\mathrm{C}$ allele was observed among American women of African ethnicity (Van Emburgh, et al; 2008). A reduced risk was also observed among African American women with the CYP1B1 CC genotype in comparison with those who had at least one G allele (Kato, et al; 2009).

The second gene to show positive association with breast cancer was COMT low production allele A carriage. Also COMT AG+AA genotypes were common in cases with positive lymphadenopathy and in premenopausal women. The same positive association was observed among American premenopausal women specially the heaviest ones (Thompson, et al;1998); Turkish premenopausal women with sporadic breast cancer (Sazci, et al; 2004); Chinese premenopausal women (Wan, et al; 2014); Brazilian women (dos Santos, et al; 2011); Chinese women (Wang, et al; 2010). However, in a meta-analysis study; it was observed that no significant association was reported in all genetic models in overall, European, Asian populations (Ding, et al; 2010); while Caucasian population had a significantly decreased risk of breast cancer (He, et al; 2012). Similarly, negative association was reported among Caucasian American women (Sigurdson, et al; 2009, Reding, et al; 2009); Portuguese women (Silva, et al; 2006); French women (Delor, et al; 2010); Slovenian women (Cerne, et al; 2011); Greenlandic Inut women (Ghisari, et al; 2014); German Postmenopausal women (MARIE-GENICA, 2009); Thai women (Sangrajrang, et al; 2009); Indian women (Syamala, et al; 2010, Naushad, et al; 2011; Syrian women (Lajin, et al; 2011). On the contrary, American women with COMT (AA) showed decreased breast cancer risk among postmenopausal females especially leanest ones (Thompson, et al; 1998, Wu, et al; 2003). 
The third association was with the CYP1A1 MspI C allele carriage. Other studies have shown similar results and found positive association in American women of African ethnicity (Taioli et al; 1999); Polish premenopausal women aged below 50 years (Ociepa-Zawal, et al; 2009); Brazilian premenopausal women (dos Santos, et al; 2011); Kazakh women (Balmukhanov, et al; 2013); On the contrary to this finding, negative association was reported in African American women (Kato, et al; 2009); German women (MARIE-GENICA, 2009); Russian women (Balmukhanov, et al; 2013); Brazilian women with sporadic breast cancer (Oliveira, et al; 2015); Thai women (Sangrajrang, et al; 2009).On the other hand, decreased risk with the rare allele was observed among Brazilian women (da Fonte de Amorim, et bal; 2002).

The least association was found with CYP 17 MspA1I C allele positive genotypes (CC+CT) were associated with increased breast cancer risk among Egyptian breast cancer women particularly within cases with negative breast feeding. Other studies have shown similar results reporting positive association with the CYP 17 MspA1I C allele carriage with the development of breast cancer. Examples include the findings reported in American postmenopausal women (Chen, et al; 2008); Brazilian postmenopausal women (dos Santos, et al; 2011); Iranian women (Hosseini, et al; 2009); Indian women younger than 40 years (Chakraborty, et al; 2007); Chinese postmenopausal females (Zhang, et al; 2009).

On the contrary to this findings, negative association was reported in some other studies in American women (Kato, et al; 2009); German women (MARIE-GENICA, 2009); Turkish women (Karakus, et al; 2015); Portuguese women (Silva, et al; 2006); Indian women (Syamala, et al; 2010); Indian women older than 40 years (Chakraborty, et al; 2007); and Thai women (Sangrajrang, et al; 2009). Reduced risk was reported in Greenlandic Inut women (Ghisari, et al; 2014).

These wide variations in genetic associations might be due to genomic diversity in subjects of different ethnicities; nonetheless it might also arise from biased selection criteria and low power studies. Although this study might also suffer some limitations related to a relatively small sample size and lack of expression analysis, we could conclude that cancer breast in Egyptian women is associated with rare alleles of estrogen biosynthesis and metabolizing genes including CYP1B1 G>C, and COMT G>A followed by CYP1A1 MspI, and CYP17 MspA1I.

Table 1:- PCR primer and conditions used for identification of studied gene polymorphisms.

\begin{tabular}{|c|c|c|c|c|c|}
\hline Polymorphism & Primers & Conditions of PCR & $\begin{array}{l}\text { PCR } \\
\text { Product }\end{array}$ & $\begin{array}{l}\text { Restriction } \\
\text { enzymes }\end{array}$ & $\begin{array}{l}\text { Fragments after } \\
\text { digestion }\end{array}$ \\
\hline $\begin{array}{l}\text { Cyp17 } \\
\text { MspA1I }\end{array}$ & 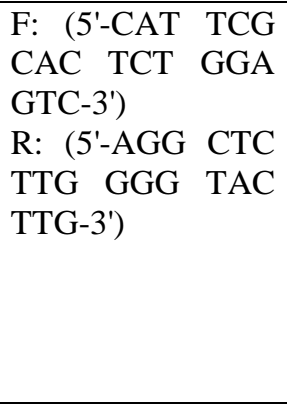 & $\begin{array}{l}\text { Denaturation at } 94^{\circ} \mathrm{C} \text { for } 5 \\
\text { min, } 30 \text { cycles at } 94^{\circ} \mathrm{C} \text { for } 1 \\
\text { min, } 58^{\circ} \mathrm{C} \text { for for } 1 \text { min and } \\
72^{0} \mathrm{C} \text { for } 1 \text { min and one final } \\
\text { cycle of extension at } 72^{\circ} \mathrm{C} \\
\text { for } 5 \mathrm{~min} \text {. }\end{array}$ & 459 bp & MspA1I & $\begin{array}{l}-459 \text { bp for TT } \\
\text { (A1A1) wild type. } \\
-335 \text { and } 124 \text { bps } \\
\text { for CC (A2A2) } \\
\text { homozygous } \\
\text { gnotype. } \\
-459,335 \text { and } 124 \\
\text { bps for TC (A1A2) } \\
\text { heterozygous } \\
\text { genotype. }\end{array}$ \\
\hline CYP1A1 MspI & $\begin{array}{l}\text { F :(5'-TAG GAG } \\
\text { TCT TGT CTC } \\
\text { ATG CCT -3') } \\
\text { R: (5'-CAG TGA } \\
\text { AGA GGT GTA } \\
\text { GCC GCT -3') }\end{array}$ & $\begin{array}{l}\text { Initial denaturation at } 95^{\circ} \mathrm{C} \\
\text { for } 10 \text { minutes, followed } \\
\text { by } 35 \text { cycles at } 95^{\circ} \mathrm{C} \text { for } \\
30 \mathrm{sec}, 55^{\circ} \mathrm{C} \text { for } 30 \mathrm{sec} \text { and } \\
72^{\circ} \mathrm{C} \text { for } 1 \text { minute and a } \\
\text { final extension of } 72^{\circ} \mathrm{C} \text { for } \\
7 \text { minutes. }\end{array}$ & $340 \mathrm{bp}$ & MspI & $\begin{array}{l}-340 \text { bp for TT wild } \\
\text { type } \\
-200 \text { and } 140 \text { bp } \\
\text { fragments for CC } \\
\text { homozygous } \\
\text { genotype } \\
-340,200,140 \mathrm{bp} \\
\text { for TC heterozygous } \\
\text { genotype. }\end{array}$ \\
\hline$C Y P 1 B 1 G>C$ & $\begin{array}{l}\text { F: (5'- TCA CTT } \\
\text { GCT TTT CTC } \\
\text { TCT CC-3') } \\
\text { R: ( 5'- AAT TTC }\end{array}$ & $\begin{array}{l}\text { by initial denaturation at } \\
95^{\circ} \mathrm{C} \text { for } 10 \text { minutes, } \\
\text { followed by } 35 \text { cycles at } \\
95^{\circ} \mathrm{C} \text { for } 1 \text { minute, } 58^{0} \mathrm{C}\end{array}$ & $650 \mathrm{bp}$ & Eco57I & $\begin{array}{l}650 \text { bp for GG wild } \\
\text { type } 340 \text { and } 310 \text { for } \\
\text { CC homozygous } \\
\text { genotype } 650, \quad 340\end{array}$ \\
\hline
\end{tabular}




\begin{tabular}{|c|c|c|c|c|c|}
\hline & $\begin{array}{l}\text { AGC TTG CCT } \\
\text { CCT G-3') }\end{array}$ & 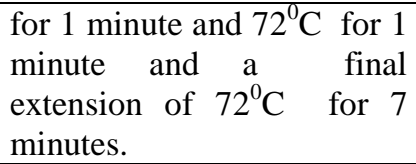 & & & $\begin{array}{l}\text { and } 310 \text { bp for } \mathrm{GC} \\
\text { heterozygous. }\end{array}$ \\
\hline$C O M T G>A$ & $\begin{array}{l}\text { F: (5'-TAC } \\
\text { GGC TAC TCA } \\
\text { GCT GTG C-3') } \\
\text { R: (5'-CTG AAC } \\
\text { GTG GTG TGA } \\
\text { ACA CC-3'). }\end{array}$ & $\begin{array}{l}94^{\circ} \mathrm{C} \text { for } 3 \text { minutes and then } \\
\text { cycled } 35 \text { times at } 94^{\circ} \mathrm{C} \text { for } \\
30 \text { seconds, } 60^{\circ} \mathrm{C} \text { for } 20 \\
\text { seconds, and } 72^{\circ} \mathrm{C} \text { for } 30 \\
\text { seconds, followed by } 72^{\circ} \mathrm{C} \\
\text { for } 5 \text { minutes. }\end{array}$ & $237 \mathrm{bp}$ & NlaIII & $\begin{array}{l}114 \text { bp for } \mathrm{G} \\
\text { allele96, } 18 \text { bp for } \mathrm{A} \\
\text { allele }\end{array}$ \\
\hline
\end{tabular}

Table 2:- Genotype frequencies of CYP17 MspA1I gene polymorphisms in a sample of Egyptian women with breast cancer compared to controls

\begin{tabular}{|l|l|l|}
\hline CYP17 T>C Genotypes & $\begin{array}{l}\text { BC cases } \\
\boldsymbol{n = 1 5 2 ( \% )}\end{array}$ & $\begin{array}{l}\text { Controls } \\
\boldsymbol{n}=\mathbf{1 0 0}(\boldsymbol{\%})\end{array}$ \\
\hline TT & $42(27.6)$ & $42(42)$ \\
\hline TC & $93(61.2)$ & $50(50)$ \\
\hline CC & $17(11.2)$ & $8(8)$ \\
\hline T Allele & $177(58.2)$ & $134(67)$ \\
\hline C Allele & $127(41.8)$ & $66(33)$ \\
\hline Statistics & $P$ & OR $(95 \%$ CI) \\
\hline CC vs. TC vs. TT & 0.058 & \\
\hline TC+CC vs. TT (Dominant) & $0.026^{*}$ & $1.9(1.07-3.34)$ \\
\hline TC vs. TT+CC (Overdominant) & 0.1 & $1.58(0.9-2.7)$ \\
\hline CC vs. TT+TC (Recessive) & 0.65 & $1.34(0.5-3.56)$ \\
\hline C Allele vs. T Allele & $0.058^{\#}$ & $1.46(1.0-2.1)$ \\
\hline $\boldsymbol{H W E}$ & $\chi^{2}=10.09, \mathrm{p}<0.05^{*}$ & $\chi^{2}=1.7, \mathrm{p}>0.05$ \\
\hline
\end{tabular}

*significant $\mathrm{p}<0.05$; \# near significant; HWE: Hardy-Weinberg Equilibrium

Table 3:- Genotype frequencies of CYP1A1 MspI gene polymorphisms in a sample of Egyptian women with breast cancer compared to controls

\begin{tabular}{|l|l|l|}
\hline CYP1A1 T>C Genotypes & $\begin{array}{l}\text { BC Cases } \\
\mathbf{n = 1 5 2 ( \% )}\end{array}$ & $\begin{array}{l}\text { Controls } \\
\mathbf{n}=\mathbf{1 0 0}(\boldsymbol{\%})\end{array}$ \\
\hline$T T$ & $66(43.4)$ & $76(76)$ \\
\hline$T C$ & $57(37.5)$ & $24(24)$ \\
\hline CC & $29(19.1)$ & $0(0)$ \\
\hline T Allele & $189(62.2 \%)$ & $176(88 \%)$ \\
\hline C Allele & $115(37.8 \%)$ & $24(22 \%)$ \\
\hline Statistics & $P$ & OR $(95 \%$ CI) \\
\hline CC vs.TC vs. TT & $<0.00001^{* *}$ & \\
\hline TC+CC vs. TT ( Dominant) & $0.000^{* * *}$ & $4.13(2.3-7.5)$ \\
\hline TC vs. CC+TT (Overdominant) & $0.035^{*}$ & $1.9(1.04-3.5)$ \\
\hline CC vs. TT+TC (Recessive) & $0.000^{* *}$ & $\mathrm{NA}$ \\
\hline C Allele vs. T Allele & $0.000^{* *}$ & $4.46(2.68-7.47)$ \\
\hline $\boldsymbol{H W E}$ & $\chi 2=6.25, \mathrm{p}<0.05$ & $\chi 2=1.86, \mathrm{p}>0.05$ \\
\hline
\end{tabular}

*significant $\mathrm{p}<0.05 ; * *$ significant $\mathrm{p}<0.001$, HWE: Hardy-Weinberg Equilibrium 
Table 4:- Genotype frequencies of CYP1B1 G>C gene polymorphisms in a sample of Egyptian women with breast cancer compared to controls

\begin{tabular}{|l|l|l|}
\hline CYP1B1 G>C Genotypes & $\begin{array}{l}\text { BC Cases } \\
\mathbf{n}=\mathbf{1 5 2}(\mathbf{\%})\end{array}$ & $\begin{array}{l}\text { Controls } \\
\mathbf{n}=\mathbf{1 0 0}(\%)\end{array}$ \\
\hline$G G$ & $41(26.9 \%)$ & $80(80 \%)$ \\
\hline$G C$ & $60(39.5 \%)$ & $20(20 \%)$ \\
\hline$C C$ & $51(33.6)$ & $0(0 \%)$ \\
\hline$G$ Allele & $142(46.7)$ & $180(90 \%)$ \\
\hline$C$ Allele & $162(53.3)$ & $20(10 \%)$ \\
\hline Statistics & $\mathrm{p}$ & OR $(95 \% \mathrm{CI})$ \\
\hline$G G$ vs. GC vs. CC & $<0.00001^{* *}$ & \\
\hline$G C+C C$ vs. GG (Dominant) & $0.000^{* *}$ & $10.8(5.66-20.87)$ \\
\hline$G C$ vs. GG+CC (Overdominant) & $0.002^{* *}$ & $2.61(1.4-4.9)$ \\
\hline$C C$ vs. GG+GC(Recessive) & $0.000^{* *}$ & $\mathrm{NA}$ \\
\hline$C$ Allele vs. G Allele & $0.000^{* *}$ & $10.26(5.98-17.8)$ \\
\hline $\boldsymbol{H W E}$ & $\chi^{2}=6.5, \mathrm{p}<0.05^{*}$ & $\chi^{2}=1.23, \mathrm{p}>0.05$ \\
\hline
\end{tabular}

*significant $\mathrm{p}<0.05 ; * *$ significant $\mathrm{p}<0.001$, HWE: Hardy-Weinberg Equilibrium

Table 5:- Genotype frequencies of COMT G>A gene polymorphisms in a sample of Egyptian women with breast cancer compared to controls

\begin{tabular}{|c|c|c|}
\hline COMT $G>A$ & $\begin{array}{c}\text { BC Cases } \\
n=152(\%)\end{array}$ & $\begin{array}{l}\text { Controls } \\
\mathrm{n}=100(\%)\end{array}$ \\
\hline \multicolumn{3}{|l|}{ Genotypes } \\
\hline$G G$ & $13(8.6)$ & 74(74) \\
\hline$G A$ & $123(80.9)$ & $26(26)$ \\
\hline$A A$ & $16(10.5)$ & $0(0)$ \\
\hline \multicolumn{3}{|l|}{ Alleles } \\
\hline$G$ & 149(49) & 173(87) \\
\hline$A$ & $155(51)$ & $27(13)$ \\
\hline Statistics & $\mathrm{p}$ & OR $(95 \% \mathrm{CI})$ \\
\hline$G G$ vs. $G A$ vs. $A A$ & $<0.00001^{* *}$ & \\
\hline$G A+A A$ vs. $G G$ ( Dominant $)$ & $0.000 * *$ & $30.43(14.0-76.4)$ \\
\hline$G A$ vs. $G G+A A$ (Overdominant $)$ & $0.000 * *$ & $12.07(6.3-23.06)$ \\
\hline$A A$ vs. $G G+G A($ Recessive $)$ & $0.002 *$ & NA \\
\hline A Allele vs. G Allele & $0.000^{* *}$ & $6.66(4.09-10.9)$ \\
\hline HWE & $\chi 2=58.25, p<0.0001 * *$ & $\chi^{2}=2.23, p>0.05$ \\
\hline
\end{tabular}

*significant $\mathrm{p}<0.05$; **significant $\mathrm{p}<0.001$, HWE: Hardy-Weinberg Equilibrium

Table 6:- Distribution of dominant model of each gene polymorphism among cases according to clinical and demographic parameters.

\begin{tabular}{|c|c|c|c|c|}
\hline Parameter & $\begin{array}{l}\text { CYP17 gene } \\
\text { CT+CC vs. TT } \\
\text { P, } \\
\text { OR(95\%CI }) \\
\end{array}$ & $\begin{array}{l}\text { CYP1A1 gene } \\
\text { CT+CC vs. TT } \\
\text { P, } \\
\text { OR(95\%CI }) \\
\end{array}$ & $\begin{array}{l}\text { CYP1B1 gene } \\
\text { GC+CC vs. GG } \\
\text { P, } \\
\text { OR }(95 \% \mathrm{CI}) \\
\end{array}$ & $\begin{array}{l}\text { COMT gene } \\
\text { GA+AA vs. GG } \\
\text { P, } \\
\text { OR(95\%CI }) \\
\end{array}$ \\
\hline $\begin{array}{l}\text { Family history } \\
\text { (+ve vs. -ve) }\end{array}$ & $\begin{array}{l}0.917, \\
1.056(0.335-3.208)\end{array}$ & $\begin{array}{l}0.139, \\
0.473(0.153-1.410)\end{array}$ & $\begin{array}{l}0.479, \\
1.426(0.474-4.198)\end{array}$ & $\begin{array}{l}0.064, \\
3.19(0.73-13.199)\end{array}$ \\
\hline $\begin{array}{l}\text { Consanguinity } \\
\text { (+ve vs. -ve) }\end{array}$ & $\begin{array}{l}0.895, \\
0.941(0.346-2.5)\end{array}$ & $\begin{array}{l}0.213 \\
0.59(0.23-1.46)\end{array}$ & $\begin{array}{l}0.182, \\
1.78(0.69-4.48)\end{array}$ & $\begin{array}{l}0.680, \\
0.721(0.104-3.765)\end{array}$ \\
\hline $\begin{array}{l}\text { Abortion } \\
\text { (+ve vs. -ve) }\end{array}$ & $\begin{array}{l}0.285 \\
0.642(0.262-1.548)\end{array}$ & $\begin{array}{l}0.281, \\
1.465(0.69-3.12)\end{array}$ & $\begin{array}{l}0.526 \\
1.28(0.56-2.94)\end{array}$ & $\begin{array}{l}0.064 \\
0.174(0.01-1.36)\end{array}$ \\
\hline $\begin{array}{l}\text { Breast feeding } \\
\text { (+ve vs. -ve) }\end{array}$ & $\begin{array}{l}0.043^{*}, \\
6.474(0.85-135.69)\end{array}$ & $\begin{array}{l}0.104, \\
0.420(0.127-1.351)\end{array}$ & $\begin{array}{l}0.851, \\
1.121(0.308-4.427)\end{array}$ & $\begin{array}{l}0.123 \\
0.344(0.073-1.809)\end{array}$ \\
\hline $\begin{array}{l}\text { Parity } \\
\text { (+ve vs. -ve) }\end{array}$ & $\begin{array}{l}0.209, \\
1.74(0.679-4.597)\end{array}$ & $\begin{array}{l}0.328, \\
0.697(0.317-1.531)\end{array}$ & $\begin{array}{l}0.359, \\
0.692(0294-1.640)\end{array}$ & $\begin{array}{l}0.703, \\
0.786(0.204-3.257)\end{array}$ \\
\hline
\end{tabular}




\begin{tabular}{|c|c|c|c|c|}
\hline $\begin{array}{l}\text { Age at menarche } \\
\text { (young vs. old) }\end{array}$ & $\begin{array}{l}0.977, \\
0.923(0.417-2.035)\end{array}$ & $\begin{array}{l}1.0, \\
1.013(0.499-2.055)\end{array}$ & $\begin{array}{l}0.798, \\
0.846(0.378-1.885)\end{array}$ & $\begin{array}{l}0.417, \\
1.893(0.534-6.792)\end{array}$ \\
\hline $\begin{array}{l}\text { Smoking } \\
\text { (+ve vs. -ve) }\end{array}$ & $\begin{array}{ll}0.168, & 0.606(0.278- \\
1.316) & \end{array}$ & $\begin{array}{l}0.270, \\
0.695(0.345-1.398)\end{array}$ & $\begin{array}{l}0.122, \\
0.567(0.259-1.240)\end{array}$ & $\begin{array}{l}0.428 \\
0.623(0.177-2.36)\end{array}$ \\
\hline $\begin{array}{l}\text { Menopause } \\
\text { (+ve vs. -ve) }\end{array}$ & $\begin{array}{l}0,224 \\
0.638(0.289-1.4)\end{array}$ & $\begin{array}{l}0.237, \\
1.476(0.736-2.965)\end{array}$ & $\begin{array}{l}0.073, \\
0.509(0.226-1.139)\end{array}$ & $\begin{array}{l}0.020 * \\
0.190(0.028-0.959)\end{array}$ \\
\hline $\begin{array}{l}\text { Estrogen receptor } \\
\text { (+ve vs. -ve) }\end{array}$ & $\begin{array}{ll}0.961, & 1.018(0.47- \\
2.24) & \\
\end{array}$ & $\begin{array}{ll}0.979, & 0.991(0.49- \\
2.01) & \\
\end{array}$ & $\begin{array}{ll}0.522, & 1.273(0.572- \\
2.848) & \end{array}$ & $\begin{array}{ll}0.111, & 0.397(0.106- \\
1.432) & \\
\end{array}$ \\
\hline $\begin{array}{l}\text { Progesterone } \\
\text { receptor } \\
(+\mathrm{ve} \text { vs. -ve) }\end{array}$ & $\begin{array}{ll}0.876, & 0.944(0.430- \\
2.077) & \end{array}$ & $\begin{array}{ll}0.986, & 1.006(0.495- \\
2.045) & \end{array}$ & $\begin{array}{ll}0.414, & 1.365(0.608- \\
3.088) & \end{array}$ & $0.374(0.10$ \\
\hline $\begin{array}{l}\text { Stage } \\
\text { Early vs. late }\end{array}$ & $\begin{array}{l}0.041^{*}, 0.289(0.086- \\
0.961)\end{array}$ & $\begin{array}{ll}0.098, & 3.405(0.84- \\
15.977) & \end{array}$ & $\begin{array}{l}0.738,1.535(0.373- \\
7.288)\end{array}$ & $\begin{array}{ll}1.0, & 1.344(0.159- \\
29.699) & \end{array}$ \\
\hline $\begin{array}{l}\text { Grade } \\
\text { Early vs. late }\end{array}$ & $\begin{array}{l}1.0,1.547(0.155- \\
37.468)\end{array}$ & $\begin{array}{l}0.763,0.5(0.056- \\
3.824)\end{array}$ & $\begin{array}{l}0.877,0.542(0.070- \\
4.843)\end{array}$ & $\begin{array}{l}0.081,0.121(0.014- \\
1.177)\end{array}$ \\
\hline $\begin{array}{l}\text { Metastasis } \\
(+ \text { ve vs. -ve) }\end{array}$ & $\begin{array}{ll}0.557, & 0.757(0.267- \\
2.076) & \\
\end{array}$ & $\begin{array}{ll}0.673, & 0.840(0.344- \\
2.031) & \\
\end{array}$ & $\begin{array}{ll}0.966, & 0.981(0.360- \\
2.612) & \end{array}$ & $0.062, \mathrm{NA}$ \\
\hline $\begin{array}{l}\text { Lymph nodes } \\
\text { (+ve vs. -ve) }\end{array}$ & $\begin{array}{ll}0.449, & 0.750(0.332- \\
1.681) & \end{array}$ & $1.23(0.603-$ & $\begin{array}{ll}0.081, & 0.499(0.21- \\
1.167) & \end{array}$ & $\begin{array}{l}0.02 * \\
0.930)\end{array}$ \\
\hline
\end{tabular}

*significant $\mathrm{p}<0.05 ; * *$ significant $\mathrm{p}<0.001$, HWE: Hardy-Weinberg Equilibrium

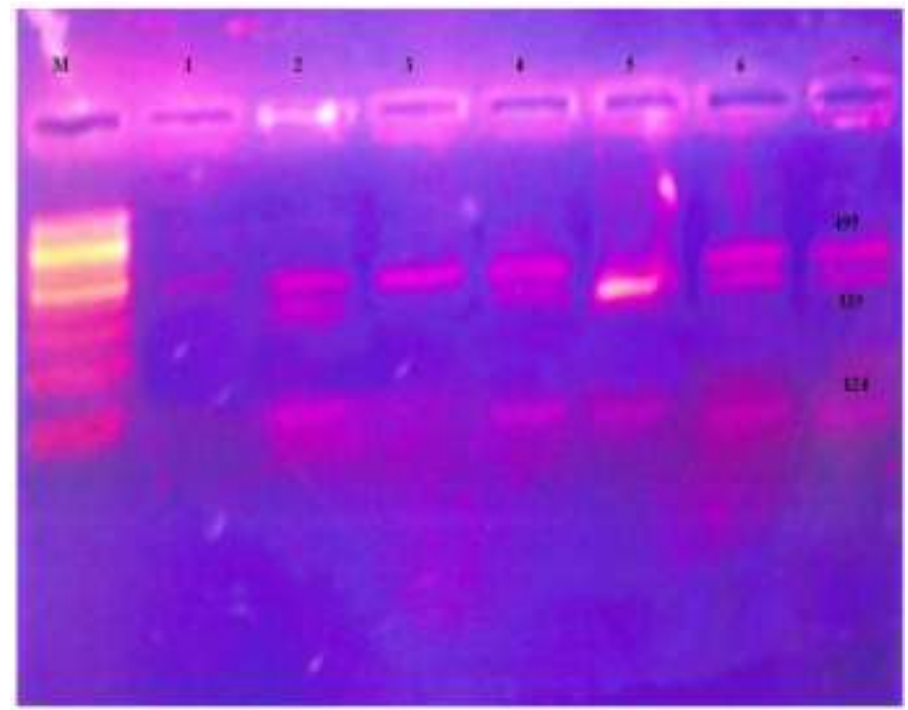

Fig1:- CYP17 MspA1I PCR product digestion using MspA1I. Lane M : 100 bp DNA ladder marker. Lane 1,3: wild TT genotype, lane 2,4,6,7 TC heterozygous genotype and lane 5: homozygous CC genotype 


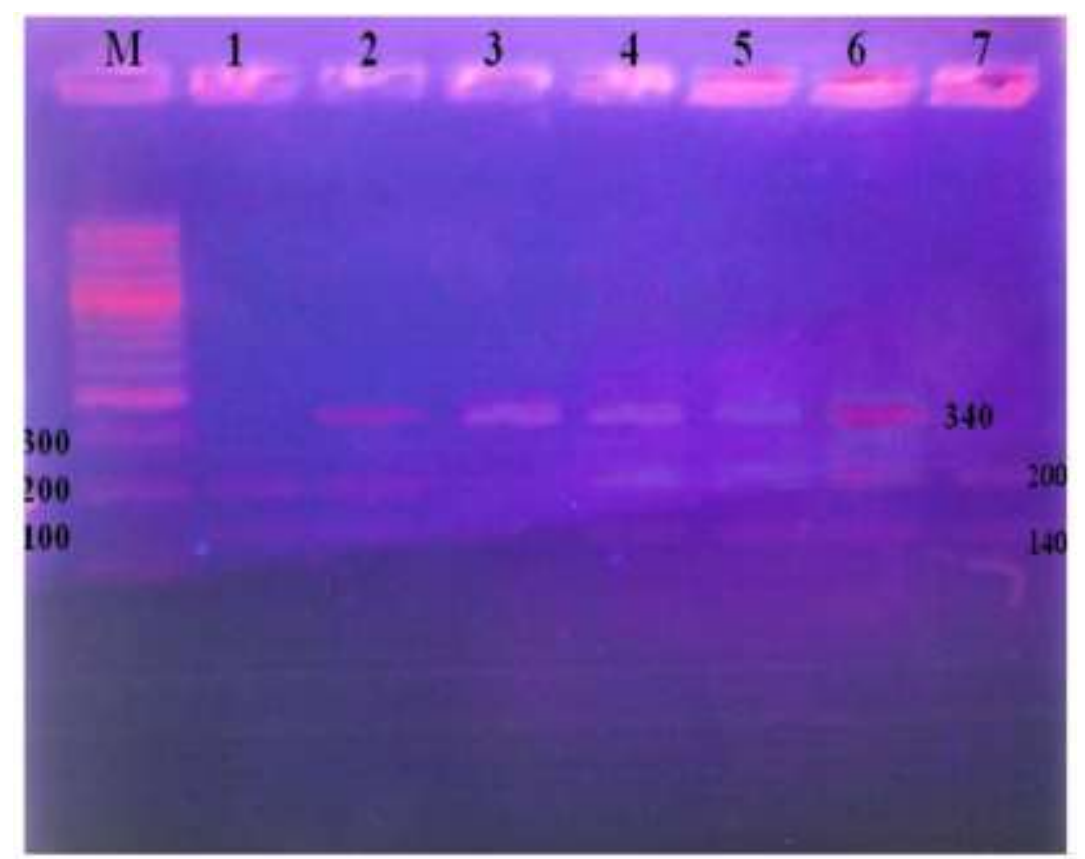

Fig 2:- CYP1A1 T>C polymorphism digestion using MspI enzyme. Lane M 100 bp DNA ladder marker. Lane 3 : wild TT genotype. Lane 2,4 - 6 : heterozygous TC genotype. Lane 1,7: homozygous mutant CC genotype.

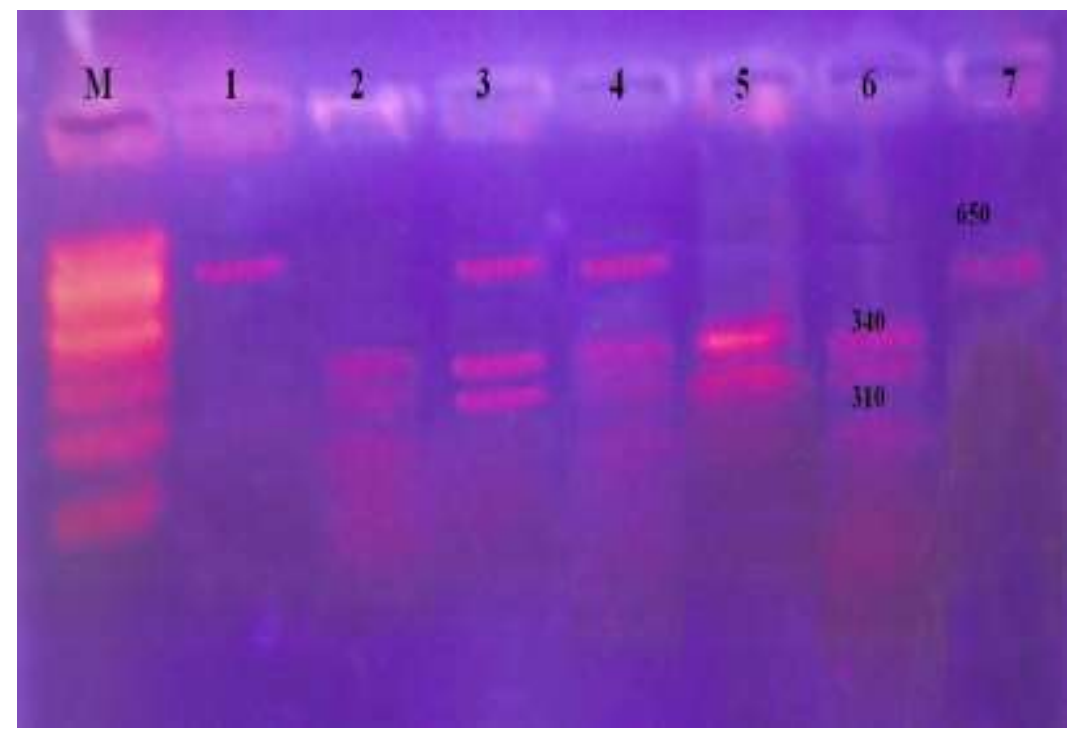

Fig 3:- PCR product digestion of CYP1B1 G>C polymorphism using ECO571 enzyme. Lane M : 100 bp DNA ladder marker. Lane 1, 7: wild type GG, lane 2,5,6 :homozygous mutant CC genotype and Lane 3,4: heterozygous GC genotype. 


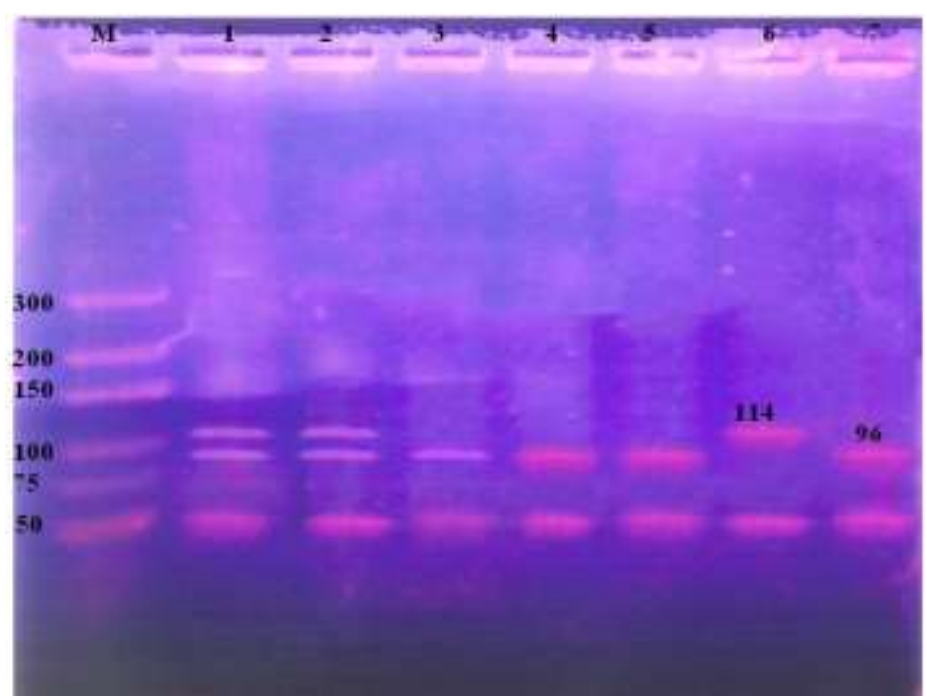

Fig 4:- PCR product digestion of COMT G>A polymorphism using NlaIII enzyme. Every digested PCR Product has 27, 42 and $54 \mathrm{bp}$ fragments. The presence of $\mathrm{G}$ allele gives fragment at $114 \mathrm{bp}$, while the presence of A allele gives 96 and 18 bp fragments. Lane M: DNA ladder marker, lane 1,2: heterozygous GA genotype, lane 6: wild type GG and lane 3-5,7 homozygous mutant AA genotype. The other fragments run out of the gel.

\section{Acknowledgement:-}

The authors are very grateful to of all cases and healthy control subjects who participated in this work, and to the team of General Surgery \& Oncology Department, Faculty of Medicine, Mansoura University for their great help and support throughout this study.

Conflict of Interest: The authors state that this study is completely free from all issues related to interest conflict.

\section{References:-}

1. Balmukhanov TS , Khanseitova AK, Nigmatova VG, Varchenko SP, Sh. Zh. Talaeva, Aitkhozhina NA . Associations of Polymorphisms of the CYP1A1 and CYP1B1 Cytochrome P450 Genes with Breast Cancer in Kazakhstan. Advances in Breast Cancer Research, 2013, 2, 51-55.

2. Bozina N, Bradamante V, Lovric M: Genetic polymorphism of metabolic enzymes P450 (CYP) as a susceptibility factor for drug response, toxicity, and cancer risk. Arh Hig Rada Toksikol 2009; 60:217-242.

3. Butterworth NJ, Dragunow M. Medial septal cholinergic neurons express c-Jun but do not undergo DNA fragmentation after fornix-fimbria transections. Brain Res Mol Brain Res 1996; 43: 1-12.

4. Carey AH, Waterworth D, Patel K, White D, Little J, Novelli P et al. Polycystic ovaries and premature male pattern baldness are associated with one allele of the steroid metabolism gene CYP17. Hum Mol Genet 1994; 3:1873-1876.

5. Cavalieri EL, Kumar S, Todorovic R, Higginbotham S, Badawi AF, Rogan EG. Imbalance of estrogen homeostasis in kidney and liver of hamsters treated with estradiol: implications for estrogen-induced initiation of renal tumors. Chem Res Toxicol 2001;14:1041-50.

6. Cavalieri EL, Stack DE, Devanesan PD, Todorovic R, Dwivedy I, Higginbotham S, Johansson SL, Patil KD, Gross ML, Gooden JK, et al.: Molecular origin of cancer: catechol estrogen-3,4-quinones as endogenous tumor initiators. Proc Natl Acad Sci USA 1997; 94(20):10937-10942.

7. Cerne JZ, Pohar-Perme M, Novakovic S, Frkovic-Grazio S, Stegel V, Gersak K. Combined effect of CYP1B1, COMT, GSTP1, and MnSOD genotypes and risk of postmenopausal breast cancer. J Gynecol Oncol 2011; 22:110-119.

8. Chakraborty A, Murthy NS, Chintamani C, Bhatnagar D, Mohil RS, Sharma PC, Saxena S. CYP17 gene polymorphism and its association with high-risk north Indian breast cancer patients. J Hum Genet 2007; 52:159-165.

9. Chen Y, Gammon MD, Teitelbaum SL, Britton JA, Terry MB, Shantakumar S et al. Estrogen-biosynthesis gene CYP17 and its interactions with reproductive, hormonal and lifestyle factors in breast cancer risk: results from the Long Island Breast Cancer Study Project. Carcinogenesis. 2008; 29:766-771. 
10. da Fonte de Amorim L, Rossini A, Mendonca G, Lotsch P, de Almeida Simão T, de Moura Gallo C, Pinto L. CYP1A1, GSTM1, and GSTT1 polymorphisms and breast cancer risk in Brazilian women. Cancer Lett 2002;181:179-86.

11. Delort L, Satih S, Kwiatkowski F, Bignon YJ, Bernard-Gallon DJ. Evaluation of breast cancer risk in a multigenic model including low penetrance genes involved in xenobiotic and estrogen metabolisms. Nutr Cancer 2010; 62:243-251.

12. Ding HX, Fu YY, Chen WX, Wang ZW. COMT Val158Met polymorphism and breast cancer risk: evidence from 26 case-control studies. Breast Cancer Res Treat 2010; 123:265-270.

13. dos Santos RA, Teixeira AC, Mayorano MB, Carrara HH, de Andrade J, Takahashi CS: Variability in estrogenmetabolizing genes and their association with genomic instability in untreated breast cancer patients and healthy women. J Biomed Biotechnol 2011; 2011:571784. doi:10.1155/2011/571784. Epub 2011 Jun 14.

14. Elatar I. Cancer registration, NCI Egypt 2001, Cairo, Egypt, National Cancer Institute,2002.

15. Fan YS, Sasi R, Lee C, et al. Localization of the humanCYP17 gene to 10q24.3 by fluorescence insitu hybridization and simultaneous chromosome banding. Genomics, 1992; 14, 1110-1.

16. Feigelson HS, Shames LS, Pike MC, Coetzee GA, Stanczyk FZ, Henderson BE. Cytochrome P450c17alpha gene (CYP17) polymorphism is associated with serum estrogen and progesterone concentrations. Cancer Res 1998; 58:585-587.

17. Fredslund SO, Bonefeld-Jorgensen EC: Breast cancer in the Arctic-changes over the past decades. Int $\mathbf{J}$ Circumpolar Health 2012; 71:19155.

18. Ghisari M, Eiberg H, Manhai Long and Eva C Bonefeld-Jørgensen1* Polymorphisms in Phase I and Phase II genes and breast cancer risk and relations to persistent organic pollutant exposure: a case-control study in Inuit women. Environmental Health 2014; 13:19.

19. He XF, Wei W, Li SX, Su J, Zhang Y, Ye H, Liu Y and Wang W. Association between the COMT Val158Met polymorphism and breast cancer risk: a meta-analysis of 30,199 cases and 38,922 controls. Mol Biol Rep. 2012 Jun; 39(6):6811-23.

20. Hosny G, Elkaffas SM. A prediction model for the incidence patterns of female breast cancers in Alexandria, Egypt. J Egypt Public Health Assoc. 2002; 77(3-4):329-45.

21. Hosseini M, Houshmand M, Ebrahimi A. Breast cancer risk not only was not associated with CYP17 A2 allele but also was related to A1 allele. Arch Med Sci 2009; 5:103-106.

22. Jiao H, Liu C, Guo W, Peng L, Chen Y and. Martin FL. Association of CYP1B1 Polymorphisms with Breast Cancer: A Case-Control Study in the Han Population in Ningxia Hui Autonomous Region, P. R. China Biomark Insights. 2010 Feb 12; 5 :21-27.

23. Karakus N, Kara N, Ulusoy AN, Ozaslan C, Tural S, Okan I. Evaluation of CYP17A1 and LEP Gene Polymorphisms in Breast Cancer. Oncol Res Treat. 2015;38(9):418-22. doi: 10.1159/000438940. Epub 2015 Aug

24. Kato I, Cichon M, Yee CL, Land S, Korczak JF. African American-preponderant single nucleotide polymorphisms (SNPs) and risk of breast cancer. Cancer Epidemiol 2009; 33:24-30.

25. Kawajiri K, Watanabe J, Gotoh O, et al. Structure and drug inducibility of the human cytochrome P-450c gene. Eur J Biochem 1986;159:219-25.

26. Kristensen VN, Borresen-Dale AL. Molecular epidemiology of breast cancer: genetic variation in steroid hormone metabolism. Mutat Res 2000;462:323 - 33.

27. Lachman HM, Papolos DF, Saito T, Yu YM, Szumlanski CL, Weinshilboum RM. Human catechol-omethyltransferase pharmacogenetics: description of a functional polymorphism and its potential application to neuropsychiatric disorders. Pharmacogenetics. 1996; 6:25-243.

28. Lajin B, Hamzeh AR, Ghabreau L, Mohamed A, Al Moustafa AE, Alachkar A: Catechol-O-methyltransferase Val 108/158 Met polymorphism and breast cancer risk: a case control study in Syria. Breast cancer (Tokyo, Japan) 2011; Epub ahead of print.

29. Law MR. Genetic predisposition to lung cancer. Br J Cancer 1990;61:195-206.

30. Liehr JG, Fang WF, Sirbasku DA, Ari-Ulubelen A: Carcinogenicity of catechol estrogens in Syrian hamsters. J Steroid Biochem 1986, 24(1):353-356.

31. MARIE-GENICA Consortium on Genetic Susceptibility for Menopausal Hormone Therapy Related Breast Cancer Risk. Genetic polymorphisms in phase I and phase II enzymes and breast cancer risk associated with menopausal hormone therapy in postmenopausal women. Breast Cancer Res Treat 2009; 119:463-474.

32. Miller WL. Early steps in androgen biosynthesis: from cholesterol to DHEA. Baillieres Clin Endocrinol Metab 1998; 12:67-81. 
33. Mitrunen K, Hirvonen A. Molecular epidemiology of sporadic breast cancer. The role of polymorphic genes involved in oestrogen biosynthesis and metabolism. Mutat Res 2003;544:9 - 41.

34. Naushad SM, Reddy CA, Rupasree Y, Pavani A, Digumarti RR, Gottumukkala SR, Kuppusamy P, Kutala VK: Cross-talk between one-carbon metabolism and xenobiotic metabolism: implications on oxidative DNA damage and susceptibility to breast cancer. Cell Biochem Biophys. 2011, 61 (3): 715-723. 10.1007/s12013-011-9245-X.

35. Ociepa-Zawal M, Rubiś B, Filas V, Bręborowicz J, Trzeciak WH. Studies on CYP1A1, CYP1B1 and CYP3A4 gene polymorphisms in breast cancer patients. Ginekol Pol. 2009 Nov; 80(11):819-23.

36. Okobia MN, Bunker CH, Garte SJ, Zmuda JM, Ezeome ER, Anyanwu SN, Uche EE, Osime U, Ojukwu J, Kuller LH, Ferrell RE, Taioli E. Cytochrome P450 1B1 Val432Leu polymorphism and breast cancer risk in Nigerian women: a case control study. Infect Agent Cancer 2009; 4 (Suppl 1):S12.

37. Oliveira CB, Cardoso-Filho C, Bossi LS, Lourenço GJ, Costa-Gurgel MS, Lima CS. Association of CYP1A1 A4889G and T6235C polymorphisms with the risk of sporadic breast cancer in Brazilian women. Clinics (Sao Paulo). 2015 Oct;70(10):680-5. doi: 10.6061/clinics/2015(10)04.

38. Reding KW, Chen C, Lowe K, Doody DR, Carlson CS, Chen CT, et al. Estrogen-related genes and their contribution to racial differences in breast cancer risk. Cancer Causes Control 2012; 23(5): 671-681.

39. Reding KW, Weiss NS, Chen C, Li CI, Carlson CS, Wilkerson HW, Farin FM, Thummel KE, Daling JR, Malone KE. Genetic polymorphisms in the catechol estrogen metabolism pathway and breast cancer risk. Cancer Epidemiol Biomarkers Prev. 2009; 18:1461-1467.

40. Sangrajrang S, Sato Y, Sakamoto H, Ohnami S, Laird NM, Khuhaprema T, Brennan P, Boffetta P, Yoshida T. Genetic polymorphisms of estrogen metabolizing enzyme and breast cancer risk in Thai women. Int J Cancer $2009 ; 125: 837-843$.

41. Sazci A, Ergul E, Utkan NZ, Canturk NZ, Kaya G. Catechol- O-methyltransferase Val 108/158 Met polymorphism in premenopausal breast cancer patients. Toxicology 2004; 204:197-202.

42. Scanlon PD, Raymond FA, Weinshilboum RM. Catechol- O-methyltransferase: thermolabile enzyme in erythrocytes of subjects homozygous for allele for low activity. Science. 1979; 5:63-65.

43. Sigurdson AJ, Bhatti P, Chang SC, Rajaraman P, Doody MM, Bowen L, Simon SL, Weinstock RM, Linet MS, Rosenstein M, Stovall M, Alexander BH, Preston DL, Struewing JP. Polymorphisms in estrogen biosynthesis and metabolism-related genes, ionizing radiation exposure, and risk of breast cancer among US radiologic technologists. Breast Cancer Res Treat 2009; 118:177-184.

44. Silva SN, Cabral MN, Bezerra de Castro G, Pires M, Azevedo AP, Manita I, Pina JE, Rueff J and Gaspar J. Breast cancer risk and polymorphisms in genes involved in metabolism of estrogens (CYP17, HSD17ß1, COMT and MnSOD): Possible protective role of MnSOD gene polymorphism Val/Ala and Ala/Ala in women that never breast fed Oncol Rep. 2006 oct; 16(4): 781-8.

45. Sun MY, Du HY, Zhu AN, Liang HY, de Garibay GR, Li FX , Li M, and Xue-Xi Yang XX. Genetic Polymorphisms in Estrogen-Related Genes and the Risk of Breast Cancer among Han Chinese Women Int. J. Mol. Sci. 2015 Feb 13; 16(2): 4121-35; doi:10.3390/ijms16024121.

46. Syamala VS, Syamala V, Sheeja VR, Kuttan R, Balakrishnan R, Ankathil R. Possible risk modification by polymorphisms of estrogen metabolizing genes in familial breast cancer susceptibility in an Indian population. Cancer Invest 2010; 28:304-311.

47. Taioli E, Bradlow HL, Garbers SV, Sepkovic DW, Osborne MP, Trachman J, Ganguly S, Garte SJ. Role of estradiol metabolism and CYP1A1 polymorphisms in breast cancer risk. Cancer Detect Prev 1999; 23:232-237.

48. Thompson PA, Shields PG, Freudenheim JL, Stone A, Vena JE, Marshall JR, Graham S, Laughlin R, Nemoto T, Kadlubar FF, Ambrosone CB . Genetic polymorphisms in catechol- O-methyltransferase, menopausal status, and breast cancer risk. Cancer Res 1998; 58:2107-2110

49. Van Emburgh BO, Hu JJ, Levine EA, Mosley LJ, Perrier ND, Freimanis RI, Allen GO, Rubin P, Sherrill GB, Shaw CS, Carey LA, Sawyer LR, Miller MS. Polymorphisms in CYP1B1,GSTM1, GSTT1 and GSTP1, and susceptibility to breast cancer. Oncol Rep 2008; 19:1311-1321

50. Wan GX1, Cao YW1, Li WQ1, Li YC1, Li F1The Catechol-O-Methyltransferase Val158Met Polymorphism Contributes to the Risk of Breast Cancer in the Chinese Population: An Updated Meta-Analysis. J Breast Cancer. 2014 Jun;17(2):149-56. doi: 10.4048/jbc.2014.17.2.149. Epub 2014 Jun 27.

51. Wang Q, Wang YP, Li JY, Yuan P, Yang F, Li H. Polymorphic catechol-O-methyltransferase gene, soy isoflavone intake and breast cancer in postmenopausal women: a case- control study. Chin J Cancer 2010; 29:683-688.

52. Wu AH, Tseng CC, Van Den Berg D, Yu MC. Tea intake, COMT genotype, and breast cancer in AsianAmerican women. Cancer Res 2003; 63:7526-7529. 
53. Yager D, Liehr JG. Molecular mechanisms of estrogen carcinogenesis. Annu Rev Pharmacol Toxicol 1996; 36:203-232.

54. Yue W, Yager JD, Wang JP, Jupe ER, Santen RJ. Estrogen receptor-dependent and independent mechanisms of breast cancer carcinogenesis. Steroids 78 (2013) 161-170.

55. Zhang L, Gu L, Qian B, Hao X, Zhang W, Wei Q, Chen K. Association of genetic polymorphisms of ER-alpha and the estradiol-synthesizing enzyme genes CYP17 and CYP19 with breast cancer risk in Chinese women. Breast Cancer Res Treat 2009; 114:327-338. 